\title{
A REMARK ON METRIC BOOLEAN RINGS
}

\section{MALCOLM F. SMILEY}

The purpose of this note is to prove that if, on a ring $B \equiv[a, b$, $c, \cdots]$ with unity element 1 , a real valued function $\mu(a)$ is defined satisfying

$$
\begin{gathered}
\mu(a)>0 \text { for every } a \neq 0, \\
\mu(a+b)+2 \mu(a b)=\mu(a)+\mu(b)
\end{gathered}
$$

for every $a, b \in B$, then $B$ is a metric Boolean ring ${ }^{1}$ [2, pp. 41 and 96]. This result is analogous to one of Glivenko's [3] which states that every metric lattice is modular $[2$, p. 42$]$. We discuss also the following modification of (1):

$$
\mu(a) \geqq 0 \quad \text { for every } a \in B .
$$

The conditions (2) and (3) also lead, via identification, to a metric Boolean ring.

THEOREM 1. Let $B$ be a ring with unity element 1 on which is defined a real valued function $\mu(a)$ satisfying (1) and (2). Then $B$ is a metric Boolean ring.

The following lemma lists the steps in our proof of Theorem 1.

Lemma 1. For every $a, b \in B$, we have (i) $\mu(a)=0$ if and only if $a=0$, (ii) $\mu(a b)=\mu(b a)$, (iii) $\mu(1+a)=\mu(1)-\mu(a)$, (iv) $\mu\left(a^{2} b\right)=\mu\left(a b^{2}\right)$, (v) $\mu\left(a^{2}\right)=\mu(a)$, (vi) $a+a=0$, (vii) $a^{2}=a$.

Proof. (i) Set $b=0$ in (2) and use (1). (ii) This is clear by (2). (iii) Set $b=1$ in (2). (iv) From (2) and (iii) we have

$$
\mu(a+b+1)+2 \mu(a b+a)=\mu(a)+\mu(1)-\mu(b) .
$$

Using (2) again gives

$$
\mu(a+b+1)+2 \mu(a b)+2 \mu(a)-4 \mu\left(a^{2} b\right)=\mu(a)+\mu(1)-\mu(b) .
$$

Rearranging, we find that

$$
4 \mu\left(a^{2} b\right)=\mu(a+b+1)+2 \mu(a b)+\mu(a)+\mu(b)-\mu(1) .
$$

Presented to the Society February 24, 1945; received by the editors January 17 , 1945.

Opinions and assertions contained in this paper are those of the author and are not to be construed as official or as reflecting the views of the United States Navy Department or of the Naval Service at large.

${ }^{1}$ Numbers enclosed in brackets denote references given at the end of the paper. 
Interchanging $a$ and $b$ in this equation yields (iv). (v) Set $b=1$ in (iv). (vi) Use (2) with $a=b$, (v), and then (i). (vii) By (2), (vi), and (iii), we have

$$
\mu\left(a^{2}+a\right)=\mu(a(a+1))=(1 / 2)[\mu(a)+\mu(a+1)-\mu(1)]=0 .
$$

Now (i) and (vi) give (vii).

The condition (vii) is Stone's definition of a Boolean ring [4]. That $B$ is a metric Boolean ring is then immediate by (1) and (2).

REMARK. The referee has pointed out that the method of proof of Lemma 1 actually yields the following theorem.

THEOREM. Let $B$ be a ring with unity element and let $R$ be a ring with unity element 1 in which $2=1+1$ is not a divisor of zero. If a function $\mu(a)$ is defined on $B$ with values in $R$, which satisfies (2) and

$$
\text { if } a \neq 0 \text {, then } \mu(a) \neq 0 \text {; }
$$

then $B$ is a Boolean ring.

We turn now to the conditions (2) and (3).

THEOREM 2. Let $B$ be a ring with unity element 1 on which is defined a real valued function $\mu(a)$ satisfying (2) and (3). Then the set $B_{0}$ of all $a \in B$ for which $\mu(a)=0$ is an ideal of $B$ and the difference ring $B-B_{0}$ is a metric Boolean ring.

Proof. First, if $a, b \in B_{0}$, then, by (2), we have

$$
\mu(a+b)+2 \mu(a b)=\mu(a)+\mu(b)=0,
$$

and consequently, by (3), $\mu(a+b)=\mu(a b)=0$. Thus $B_{0}$ is a subring of $B$. To show that $B_{0}$ is an ideal, we first note that (ii) and (iii) of Lemma 1 are still valid. Now, using (2) and (iii) of Lemma 1, we obtain for $a \in B_{0}, c \in B$,

$$
\begin{aligned}
2 \mu(a(1+c)) & =\mu(a)+\mu(1+c)-\mu(1+a+c), \\
& =\mu(1)-\mu(c)-\mu(1)+\mu(a+c), \\
& =-\mu(c)+\mu(a)+\mu(c)-2 \mu(a c), \\
& =-2 \mu(a c) .
\end{aligned}
$$

Hence, by (3), $\mu(a c)=0$, and (ii) of Lemma 1 yields also $\mu(c a)=0$. We conclude that $B_{0}$ is an ideal of $B$. Note also that if $a \in B_{0}, c \in B$, we have

$$
\mu(a+c)=\mu(a)+\mu(c)-2 \mu(a c)=\mu(c) .
$$


Thus the function $\mu(a)$ is constant on each element of $B-B_{0}$. The conclusion of Theorem 2 is now clear by Theorem 1 .

Note added in proof. The assumption of a unity element for $B$ made in Theorems 1 and 2 may be avoided as follows. Expand $\mu(a+b+c)$ as in the proof of (iv) of Lemma 1 and interchange $a$ and $b$ to obtain (4): $\mu(a b a c)=\mu(b a b c)$. Setting $b=c=-a$ then gives $\mu\left(a^{4}\right)=\mu\left(-a^{4}\right)$. Putting $b=a$ in (2) yields, via (3), $\mu(a) \geqq \mu\left(a^{2}\right)$, and hence also $\mu(-a) \geqq \mu\left(a^{2}\right)$. With $b=-a$ in (2), we obtain $\mu(a)+\mu(-a)=2 \mu\left(-a^{2}\right)$. Thus we have $2 \mu\left(-a^{4}\right)=\mu\left(a^{2}\right)+\mu\left(-a^{2}\right) \geqq 2 \mu\left(a^{4}\right)=2 \mu\left(-a^{4}\right)$, and consequently $2 \mu\left(a^{4}\right)=2 \mu\left(a^{2}\right)=2 \mu\left(-a^{2}\right)=\mu(a)+\mu(-a)$, from which (5): $\mu\left(a^{2}\right)=\mu(a)=\mu(-a)$ follows. From (4) and (5) we obtain (6): $\mu\left(a b a^{2}\right)=\mu(b a b a)=\mu(b a)$ and also $\mu\left(a^{6}\right)=\mu\left(a^{8}\right)=\mu\left(a^{8}\right)=\mu(a)$. Now (2) gives $\mu\left(a+a^{2}\right)=0$. To show that $B_{0}$ of Theorem 2 is an ideal we have, for $a \in B_{0}, b \in B, \mu\left(a^{2}+a b\right)=\mu\left(a^{2}\right)+\mu(a b)-2 \mu\left(a^{8} b\right)=-\mu(a b)$, by (5), (6), and (ii).

On the other hand, the assumption of the unity element for $B$ in the referee's theorem of our Remark is essential. For we may take $R$ to be the ring of integers modulo an odd integer $m$, the elements and addition of $B$ to be those of $R$, and define $a b \equiv 0, \mu(a) \equiv a$ for every $a, b \in B$. All the hypotheses of the theorem of our Remark, except that $B$ have a unity element, are valid, but $B$ is no Boolean ring. The standard process of introducing a unity element $[1$, p. 23] thus cannot preserve $\left(1^{\prime}\right)$ and (2).

\section{REFERENCES}

1. A. A. Albert, Modern higher algebra, Chicago, 1937.

2. Garrett Birkhoff, Lattice theory, Amer. Math. Soc. Colloquium Publications, vol. $25,1940$.

3. V. Glivenko, Gémétrie des systèmes de choses normées, Amer. J. Math. vol. 58 (1936) pp. 799-828.

4. M. H. Stone, The theory of representations for Boolean algebras, Trans. Amer. Math. Soc. vol. 40 (1936) pp. 37-111.

United States Naval Academy, Postgraduate School 